\title{
La técnica Alexader y John Dewey: propuesta para una educación corporal
}

\author{
The Alexander Technique and John Dewey: \\ Proposal for a Corporal Education
}

GLORIA LUQUE MOYA*

\begin{abstract}
Resumen: La reflexión filosófica sobre el cuerpo ha experimentado un auge en los últimos siglos, tratando de superar desde diferentes aproximaciones la devaluación, abandono e incluso rechazo del mismo. En este sentido, estas páginas revisan el método de educación corporal desarrollado por el actor australiano Frederick Matthias Alexander en relación con el filósofo americano John Dewey y su propuesta filosófica. Alexander influenció la vida y obra de Dewey, proporcionándole no sólo una crítica sobre los dualismos construidos en torno al cuerpo, sino también una técnica corporal desde la cual superarlos.
\end{abstract}

Palabras claves: cuerpo, mente, experiencia, Técnica Alexander.

\begin{abstract}
The philosophical reflection about body has increased in the recent centuries, trying to overcome devaluation, neglect and rejection of the body from different approaches. These pages review the method of corporal education developed by the Australian actor Frederick Matthias Alexander in relation to the American philosopher John Dewey and his philosophical proposal. Alexander influenced the life and work of Dewey, providing not only a criticism of the dualisms built around the body, but a technique from which to overcome them.
\end{abstract}

Keywords: Body, Mind, Experience, Alexander Technique.

A lo largo del siglo XX y XXI el discurso filosófico ha desarrollado nuevas vías de reflexión sobre el cuerpo, con figuras reconocidas como Merleau-Ponty o Foucault, o sub-disciplinas que tratan de reivindicar su cultivo y cuidado, como la somaestética. Estas nuevas corrientes intentan superar la devaluación producida en torno al cuerpo, que había

Fecha de recepción: 06/06/2016. Fecha de aceptación: 12/09/2016.

* Doctoranda de la Universidad de Málaga (España), Facultad de Filosofía, bajo la dirección de Luis Enrique de Santiago Guervós y María Rosa Fernández Gómez, con la tesis John Dewey, Confucio y el arte de vivir: Un estudio comparado sobre la noción de experiencia estética. Correo electrónico: glorialm@uma.es Contratada predoctoral del programa de becas de Formación del Profesorado Universitario del Ministerio de Educación. Líneas de investigación: La filosofía de John Dewey y el pragmatismo americano; estética comparada; la filosofía de Confucio. Algunas publicaciones recientes: "On Birds, Beast and Human Beings. An Approach to the Continuity between Art and Life", en E. Chudoba y C. Wilkoszewska (eds.), Naturalizing Aesthetics, Krakow, Libron, 2015, pp. 115-124; "The Pace of Aesthetic Process: A Comparative Approach" en A. Nolasco y A. R. Ferreira, Seminar Proceedings International Colloquium Creative Processes in Art, Lisbon: Centro de Investigação e Estudos em Belas-Artes (CIEBA), 2014, pp. 115-128. 
propiciado un abandono y rechazo de todo aquello que procediera del mismo. En este contexto, resulta interesante actualizar el pensamiento de John Dewey sobre el cuerpo en relación a la técnica Alexander. Como han destacado Jo Ann Boydston y Richard Shusterman ${ }^{1}$, Frederick Matthias Alexander tuvo una gran influencia en el pensamiento del filósofo americano. Así lo reconoció el propio Dewey en su correspondencia ${ }^{2}$ y en su obra Experience and Nature ${ }^{3}$ donde destaca el valor del actor y creador de la técnica en su contexto histórico social.

Ahora bien, la valía de su práctica no solo tuvo influencia en el pasado siglo, sino que hoy en día su aplicación se extiende por el mundo por los beneficios que apareja. En este sentido, estas páginas revisan la propuesta de Alexander en relación a la filosofía deweyana, tratando de destacar su actual potencial. Esta exploración, por tanto, proporciona una revisión del cuerpo a través de esta vía educativa que no sólo crítica los esquemas filosóficos modernos, sino que proporciona una filosofía meliorativa de nuestra corporalidad. Para ello, en primer lugar consideraré, a grandes rasgos, en qué consiste la técnica Alexander. En segundo lugar, atenderé a la relación de John Dewey y Frederick Matthias Alexander, estableciendo los elementos que el filósofo introduce en el último periodo de su pensamiento. En tercer lugar, a modo de conclusión, apuntaré la actualidad de esta propuesta educativa en el discurso filosófico, pero también en nuestra cotidianeidad.

\section{La técnica Alexander}

Frederick Matthias Alexander (1869-1955) fue un actor australiano que creó una metodología para propiciar una conciencia de nuestros hábitos corporales. Alexander la desarrolló cuando empezó a sufrir afonía durante sus actuaciones. Ante la repentina pérdida de voz, el actor decidió ponerse frente al espejo para observar cuál era el motivo de dicha afonía, comprobando que había desarrollado hábitos inconscientes. Esto es, el actor adquirió conciencia corporal, atisbando los hábitos asumidos y poniendo atención en los mismos para inhibirlos y evitarlos ${ }^{4}$.

1 Boydston, J. A., "John Dewey and Alexander Technique”, presentada en International Congress of Teachers of the Alexander Technique, 1986,. Disponible en http://www.alexandercenter.com/jd/deweyalexanderboydston. html [fecha de última consulta 1 de junio de 2016]; Shusterman, R., «Reedeming Somatic Reflection: John Dewey's Philosophy of Body-Mind», en Body Consciousness: A Philosophy of Mindfulness, Cambridge, Cambridge University Press, 2008, pp. 180-216.

2 Hickman, L. A. (ed.), The Correspondence of John Dewey, InteLex, versión electrónica, 1992, Carta de Dewey a Alexander del día 20 de noviembre de 1917.

3 Dewey, J., Experience and Nature, The Later Works of John Dewey, vol. I, J. Ann Boydston (ed.), Carbondale, Southern Illinois Press, 2008, capítulo VII "Nature, Life and Body-Mind, cita 2, p. 225; y capítulo VIII "Existente, Ideas and Consciousness", p. 229. En adelante las referencias a los escritos de John Dewey se basarán en la edición crítica de las obras completas publicada por Southern Illinois University: EW (The Early Works), MW (The Middle Works) y LW (The Later Works). Las citas seguirán el modelo normalizado entre los estudiosos de la obra de Dewey: la inicial de las series, seguida por el volumen y el número de la página.

4 "The Life and Discovery of F.M. Alexander", Fragmentos de McCullough, C. P., The Alexander Technique and the String Pedagogy of Paul Rolland, Arizona, Arizona State University, 1996. Disponible en http://www. alexandercenter.com/pa/stringsii.html [fecha de última consulta 1 de junio de 2016]. 
De este modo, Alexander elaboró un método educativo que buscaba superar los hábitos incorporados inconscientemente y propiciar una integridad psicofísica que permitiera una interacción equilibrada con su medio. Deseoso de presentar su descubrimiento, el actor australiano comenzó a transmitir su experiencia, proponiendo una técnica corporal que no incurriera en desvíos terminológicos, sino que enfatizara en su despliegue práctico, mostrando los aspectos claves para mejorar nuestra salud psico-corporal. Alexander decidió mudarse a Londres en 1904 para promover su técnica y crear un programa de entrenamiento. Cuando estalló la Primera Guerra Mundial se traslada a Nueva York donde enseña nueve horas al día ${ }^{5}$. En 1924, tras regresar a Inglaterra, fundó una escuela para niños en su centro pedagógico de Londres. Sin embargo, el estallido de la Segunda Guerra Mundial propicia la evacuación de la escuela a América, estableciéndose, finalmente, en Massachussets ${ }^{6}$.

Al regresar a Inglaterra en 1943, el actor es llevado a juicio por los ataques sobre su trabajo que realizó el doctor Ernst Jokl en Sudáfrica. Durante el juicio, a finales de 1947, sufrió un accidente y a la semana una apoplejía que le hizo perder el uso de su brazo y pierna izquierda. Ese año tenía 79 años, pero continuó impartiendo clase durante casi ocho años más, hasta su muerte a la edad de 86 años ${ }^{7}$. Alexander creía firmemente que su técnica abría una nueva vía para el futuro ${ }^{8}$ y quería que se convirtiera en una profesión médica. Como ha destacado Jennifer Tarr ${ }^{9}$, Alexander en su último libro intenta relacionar su técnica con la biomedicina:

Ever since I first started taking pupils, medical men have been sending me their patients because they believed that I had evolved a sound technique. I am deeply indebted to them for their encouragement and support, and especifically for the effort they are now making to bring a knowledge of my technique to the notice of those who are responsible for determining the range and nature of the medical curriculum with the aim of its being included in medical training ${ }^{10}$.

Ahora bien, ¿qué es la técnica Alexander y en qué consiste? Se trata de una forma de trabajo corporal que enseña a los alumnos a usar eficientemente sus cuerpos en el movimiento cotidiano. Normalmente, la técnica consta de varias sesiones que pueden durar entre media hora y cuarenta y cinco minutos, en las cuales el profesor trata de hacer consciente al alumno de los hábitos que padece, mediante diferentes actividades ${ }^{11}$. Alexander parte de la premisa de que la conducta humana es principalmente movimiento: levantarse, sentarse, caminar, incorporarse, abrir y cerrar cajones, entrar o salir del coche, etc.; todas estas y muchas otras

5 Maisel, E. (comp.), La técnica Alexander, Barcelona, Paidós, 1996, p. 26.

6 Ibid., p. 30.

7 Ibid., p. 31.

8 "Mi experiencia puede llegar a reconocerse algún día como un poste indicador que dirija al explorador hacia un territorio ahora "inexplorado"" [Maisel, E. (comp.), op. cit., p. 13].

9 Tarr, J., "Educating with the hands: working on the body/self in Alexander Technique", en C. McFarlane, R. L. Cohen y S. Nettleton, Sociology of Health and Illness Monographs: Body Work in Health and Social Care: Critical Themes, New Agendas, Malden, John Wiley \& Sons, 2011, p. 81.

10 Alexander, F. M., The Universal Constant in Living, London, Mouritz, 2000, p. 13.

11 Tarr, J., op. cit., p. 173. 
actividades habituales implican patrones de movimientos que construyen nuestro carácter $(s e l f)^{12}$. La técnica busca, pues, mejorar nuestra interacción y aportar un nuevo enfoque para las actividades cotidianas, las cuales solemos realizar a partir de hábitos que consideramos buenos y fiables, pero que generan usos forzados del cuerpo.

En 1907 Alexander ya propuso como premisa de partida para la práctica de esta técnica el máximo alargamiento posible de la columna vertebral, al margen de la actividad que estuviéramos realizando. Dicho alargamiento lo llamó "el verdadero y primario movimiento en todos y cada uno de nuestros actos"13. No obstante, Alexander nunca fue demasiado claro sobre la técnica por la problemática de presentarla en lenguaje verbal. Edward Maisel destaca un triple patrón en la introducción a la selección de textos traducidos al español:

1. Dejar el cuello libre (lo que significa sencillamente tratar de que no se incremente la tensión del cuello en ningún acto).

2. Dejar que la cabeza vaya hacia adelante y arriba (lo que significa sencillamente tratar de no tensar los músculos del cuello tirando de la cabeza hacia atrás o hacia abajo en ningún acto).

3. Dejar que el torso se alargue y ensanche (lo que significa sencillamente tratar de no acortar y estrechar la espalda arqueando la columna) ${ }^{14}$.

Ahora bien, la metodología de Alexander no consiste en la asimilación de una posición concreta o la transmisión de una técnica predefinida, sino que implica la facultad que todos los seres humanos poseen para hacerse conscientes de su propia corporalidad. Alexander, en este respecto, desarrolló un medio para transmitir la experiencia cinestésica, sin recurrir a una exposición verbal, que aún hoy se sigue utilizando. En primer lugar se solicita al alumno que realice cualquier actividad y seguidamente se le ordena que no la haga. Por ejemplo si se le pide que se levante, cuando este va a hacerlo, el alumno se ha de detener y tratar de percibir los hábitos sutiles que se activan cuando vamos a realizar dicho movimiento. Así, se propone una forma de cooperación en la que el profesor pone de pie, sienta o hace caminar al alumno propiciando una conciencia de los hábitos incorporados a estas acciones y promoviendo una relación armónica de cabeza-cuello-torso ${ }^{15}$.

La respiración también será un aspecto esencial dentro de este proceso de aprendizaje. Alexander otorga una atención especial a la respiración, centrando el trabajo del alumno en que esta se despliegue de manera libre. Las posibilidades de organización de movimientos durante la actividad son infinitas y la respiración será un elemento clave dentro de la misma. Por ello, habrá que potenciar una respiración consciente que este en consonancia con las actividades que estamos realizando. Esto es, la respiración se presentará como una función más del uso adecuado de uno mismo, en la que tendremos que reeducarnos. Alexander dirá

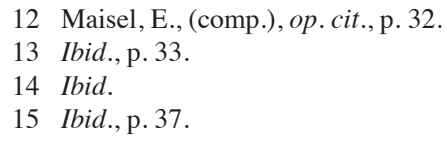


It is essential to have a proper mental attitude towards respiratory education or reeucation, and the specific acts which constitute the exercises embodied in it, together with a proper knowledge and practical employment of the true primary movement in each and every act. ${ }^{16}$

Esta técnica implica, pues, un proceso gradual por el que el alumno irá desarrollando un patrón personal para realizar sus actividades. El aprendiz de la técnica tendrá que conseguir el control y el desarrollo máximo en la respiración por su propio esfuerzo y con ayuda de un profesor. El objetivo de estas lecciones no será el aprendizaje o imitación de modelos, sino el auto-descubrimiento, aquel que nos conduzca a un aumento de la conciencia y el control propio. Esto no es tarea sencilla pues el defecto que caracterizaba al individuo en la época de Alexander, así como en la nuestra, era la mente distraída. Para el actor, ésta era una lacra común que dificultaba nuestra interacción cotidiana.

La gravedad de la incapacidad del ser humano para mantener su mente en lo que está haciendo es un aspecto ampliamente reconocido. Uno de los problemas más citados en los estudios sobre educación hablan de la curva de atención y la facilidad con la que los alumnos se desconcentran en el aula. Ante esto, se ha enfatizado en tácticas para captar la atención del alumnado; sin embargo, esta técnica va a poner el énfasis no tanto en captar pupilos, sino en que estos aprendan a desarrollar la concentración basada en la unidad mente-cuerpo.

\section{La técnica Alexander y la filosofía de John Dewey}

Dewey tuvo su primer encuentro con Alexander en 1916, y a partir de ese momento él mismo, junto con toda su familia, comenzaron a practicar dicha técnica ${ }^{17}$. En este sentido, lo que Alexander aportó a Dewey, no fue tanto una nueva restauración o noción cuerpomente, sino un mecanismo o técnica desde la cual adquirir conciencia de esta unidad. La introducción que el mismo Dewey realiza a la segunda edición de la obra en $1918^{18}$ pone de manifiesto la admiración e importancia que Alexander tuvo en el desarrollo del pensamiento deweyano, no sólo por haber alcanzado los significados, peligros y posibilidades de los cambios en los que se encontraban inmersas las personas de su época, sino por haber propuesto una metodología desde la cual corregir dichos problemas.

Para Dewey, Alexander acierta al exponer la crisis de la salud física y moral de los individuos como aquella generada por el conflicto entre las funciones del cerebro y sistema nervioso, por un lado, y las funciones de la digestión, circulación, respiración y sistema muscular por el otro ${ }^{19}$; esto es, las funciones psíquicas y físicas. La dificultad que Alexander presenta a esta escisión, y que él mismo sufrió cuando experimentó su problema de afonía, es que ante incidentes o episodios problemáticos de nuestra vida el modo habitual para tratarlos los consideraba como accidentes aislados que podían ser superados desde el aislamiento.

16 Alexander, F. M., Man's Supreme Inheritance, New York, E.P. Dutton and Company, 1918, p. 324.

17 Hickman, L. A. (ed.), op.cit., carta de Alexander a Dewey el día 9 de diciembre de 1918.

18 Dewey, J., "Introductory Words", en Alexander, F. M., Man's Supreme Inheritance, New York, E. P. Dutton and Co. 1918. En adelante citaré esta obra siguiendo la edición recogida en MW 11:350-352.

19 MW 11:350. 
Dewey, de acuerdo con Alexander, destaca los errores de los métodos paliativos que, en lugar de curar, en numerosas ocasiones lo que hacían era contribuir a un mayor desajuste en el organismo. Esto se debía a los intentos específicos y limitados que se empleaban para curar los órganos descoordinados que, en la mayor parte de los casos, ejercía un mayor mecanismo de descoordinación. En palabras de Dewey:

When the organs through which any structure, be it physiological, mental or social, are out of balance, when they are uncoordinated, specific and limited attempts at a cure only exercise the already disordered mechanism. In 'improving' one organic structure, they produce a compensatory maladjustment, usually more subtle and more difficult to deal with, somewhere else. ${ }^{20}$

Por ello, el ensayo de Alexander, lo presenta como un libro educativo que pretende dar una respuesta que no privilegie una parte del organismo sobre otra. Esto no implica el desarrollo del poder de la inteligencia; es decir, un control positivo de las funciones mediante el intelecto. La técnica consiste en una postura basada en el conocimiento de nosotros como organismo, en el que se asume esa unión y se reconoce el cuerpo como la estructura más maravillosa del universo ${ }^{21}$.

Dewey critica la tradicional asociación de la inteligencia con las formas cultivadas del hombre realizadas desde la inteligencia. Según Dewey, la técnica Alexander supera esta oposición y vuelve a unir esos dos elementos que nunca debieron ser separados. Alexander usa los términos "hábito físico" o "hábito corporal" y "hábito mental", para denominar el uso equivocado del aparato sensorio-motor y la concepción errónea sobre el organismo físico-psíquico. Él expone como estos hábitos se habían convertidos en fijos e incontrolables causando una dualidad en el hombre, no sólo a nivel de discusiones teóricas y filosóficas, sino también en la propia conciencia de su cuerpo en la vida cotidiana, generando problemas psicosomáticos.

the subject can hardly fall to cultivate a wrong mental attitude toward life in general and toward the art of living (evolving satisfactorily)", and, unable to distinguish between conscious and subconscious actions, he "suffers from various forms of mental and physical delusions, notably with regard to the physical acts he perfoms ${ }^{22}$

En contraste, Alexander mantiene que mente y cuerpo no son entidades separadas, sino funciones continuas que afectan la una a la otra recíprocamente ${ }^{23}$. Dewey cree que el valor de esta propuesta educativa radica en la reintegración de las funciones que tan desastrosamente se habían dividido y que habían dado primacía a la inteligencia sobre el

20 MW 11:350.

21 Literalmente Dewey dirá: "the most wonderful of all the structures of the vast universe" [MW 11:351].

22 Alexander, F. M., op. cit. (1918), p. 185.

23 Véase el capítulo dedicado a este tema, donde Alexander expone casos concretos, la forma en que se ha procedido y los problemas derivados por el tratamiento surgido por esa escisión de lo físico y lo psíquico, Alexander, F. M., "Habits of Thought and of Body", op. cit. (1918), pp. 73-107. 
cuerpo. Como pone de manifiesto en la respuesta que da a la crítica que Randolph Bourne ${ }^{24}$ realiza a la obra de Alexander:

Certainly one of the prime effects of acquaintance with the methods of 'conscious control' is to make one realize the superficial and over-hasty character of the methods upon which we are relying, and the corresponding importance of a fundamental method of education, one which in the course of show generations will integrate into harmonious coordinaiton our animal inheritance and our distinctively human capacities of intelligence ${ }^{25}$.

Para Dewey, ya en el periodo final de su vida, y concretamente a partir de su obra Experience and Nature (1925) el organismo, formado por la mente y el cuerpo, es algo que debe ser admitido sin recurrir a ningún tipo de escisión dualista ${ }^{26}$. La unidad físicopsíquica adquiere mayor relevancia en el capítulo 7, titulado "Nature, Life and BodyMind", donde Dewey introduce su particular noción de organismo en contraposición a la tradición filosófica. En él realiza una genealogía que comienza en el periodo clásico, analizando la relación que establecieron los griegos entre mente, naturaleza y cuerpo. Según Dewey, los primeros filósofos propusieron una relación basada en la dualidad almacuerpo y construida bajo el influjo de lo supernatural. Esta aproximación fue asimilada posteriormente por el cristianismo, llegando a convertirse en un problema formalista e irreal con la tradición moderna. Frente a esta tradición, Dewey carcteriza el cuerpo y la mente como estructuras del organismo vivo, diferenciadas por su función, pero en continua interrelación. La mente se encuentra en conexión con el cuerpo y cada cuerpo existe en un medio natural con el que sostiene conexiones de adaptación, a través de los procesos organizativos de la mente ${ }^{27}$.

Dewey va a destacar cómo la mente se desarrolla teniendo presente el pasado y el futuro, así como las estructuras biológicas del organismo y el medio. Es decir, presenta la noción de organismo desde esa continuidad cuerpo-mente en relación con la naturaleza. En este sentido, el organismo no es una estructura, sino un modo característico de interactuar el cual no es simultaneo, sino serial, se despliega en el tiempo ${ }^{28}$. Así, no podemos separar a la criatura viva en cuerpo y mente como tampoco podemos escindir organismo y naturaleza. Dewey propone una noción o modelo de coordinación orgánica entre el cuerpo y la mente, que expondrá detalladamente en el artículo publicado en Bulletin of the New York Academy of Medicine en 1928 "Body and Mind".

24 Randolph Bourne publicó una crítica a Man's Supreme Inheritance en la revista New Republic 15 (1918) que el propio Dewey respondió. Para las citas de la replica que Dewey realiza, utilizaré la recogida en MW $11: 353-355$.

25 MW 11: 355.

26 LW 1:203.

27 LW 1:212.

28 LW 1:220. 
Para Dewey, la filosofía, la ciencia y las artes (incluyendo la medicina) en un tiempo pasado estaban unidas, surgiendo las dos primeras a partir de la última ${ }^{29}$. Dewey recurre a la palabra techné griega que designaba ciencia y arte para mostrar esa unidad y presenta la escuela de Hipócrates como aquella que supo unir arte, ciencia y filosofía glorificando dicha noción. Hipócrates y su escuela tomaron prestado de Heráclito, Empedocles y Pitágoras sus términos e insistieron en la armonía de todos los elementos como condición para mantener y restaurar la salud ${ }^{30}$. Frente a esta idea de armonía, Dewey destaca los efectos desastrosos de su tiempo derivados de la división entre mente y cuerpo y apunta la necesidad de restaurar esa unidad como un todo.

The evils which we suffer in education, in religión -for example the fundamentalist attack about the evolution of men rests upon the idea of complete separation of mind and body-in the materialism of business and the aloofness of intellectuals from life, the whole separation of knowledge and practice: -all testify to the necessity of seeing mind-body as an integral whole..$^{31}$

El filósofo americano advierte que en el pensamiento occidental a diferencia de otras tradiciones ${ }^{32}$, no tenemos una noción para denominar la unidad mente-cuerpo. Por ello, aún cuando se hablan de sus relaciones, continuamos manteniendo esa división perpetua; pese a iniciar una discusión para rechazar esta escisión, permanece esta oposición. Frente a ello, Dewey propone la consideración de estas nociones desde la unidad de la acción. Esto es, según el autor, el hábito que divide lo mental y lo físico en entes separados tiene sus raíces en la consideración de ambas partes como sustancias en lugar de funciones y cualidades de la acción ${ }^{33}$.

Dewey lo ejemplifica con el acto de comer: uno puede comer porque sienta necesidad de comer, porque esté triste o porque esté alegre; comer es, asimismo, un acto social y su temperamento emocional dificulta su consideración como un acto meramente físico. Comer pan y beber vino, dirá Dewey, puede tener implícito actitudes mentales de personas que están asumiendo el aspecto sacramental y espiritual. En este sentido, podemos extraer dos consecuencias claras: que no hay necesidad de establecer líneas que separen unas funciones de otras y que hasta la acción más cotidiana y común de nuestro día a día esta cargada de potencial creador $^{34}$.

29 "It was once their aspiration to find their issue in arts; the sciences in arts of the special branches of life and philosophy in the comprehensive arto f the wise conducto of life as a whole". ["Body and Mind", LW 3:25].

30 LW 3:26.

31 LW 3:27.

32 Téngase en cuenta, por ejemplo, la noción china de mente-corazón (心) en la que no existe escisión alguna, sino que el caracter encierra ambos significados o connotaciones.

33 LW 3:28.

34 En relación a este punto, autores contemporáneos han pasado a reivindicar un nuevo estilo de vida a través de algo tan cotidiano y que muestra esa unidad cuerpo-mente como es la comida a través del concepto de slow food. Carlo Petrini, creador de este movimiento, reivindica la dignidad cultural en relación a la comida y los modos de alimentación ligados a un territorio, promoviendo una "calidad de vida" diferente al ritmo que nuestra sociedad cotidiana nos empuja. Veáse Petrini C., Slow Food, The case for Taste, New York, Columbia University Press, 2001. 
De este modo, la integración mente-cuerpo ${ }^{35}$ en la acción es, para Dewey, la cuestión más práctica que podemos pedir a nuestra civilización ${ }^{36}$. Lo más destacable respecto a esta demanda es que no se trata de una mera cuestión especulativa, y la técnica Alexander no sólo le ha proporcionado un ámbito práctico de acción sino un método desde el cual restaurar esa continuidad. Al introducir el término criatura viva en el periodo final, Dewey trata de establecer que no somos mente o cuerpo, sino organismos vivos conformados por esa estructura psicofísica que esta en continua interacción con su medio. En este sentido, la reflexión deweyana tiene cabida en nuestros días, cuando cada vez adquiere mayor importancia el cuidado y cultivo del cuerpo para una mejora en la salud corporal y psíquica.

\section{Conclusión}

El tratamiento que ha recibido el cuerpo dentro de la filosofía ha venido caracterizado en numerosas ocasiones por una devaluación en oposición a la razón, la mente o la inteligencia. Esta escisión no sólo contraponía dos aspectos del ser humano, sino que también discriminaba las actividades humanas por ser superiores (reflexión, pensamiento) o inferiores (actividades realizadas principalmente por el cuerpo que se las designa automáticas o instintivas). Sin embargo, la corriente pragmatista clásica, sobretodo a partir de William James, pone el énfasis en la unidad.

Dewey, va a tratar de superar este dualismo siguiendo la línea abierta por William James. Si bien, será la técnica Alexander la que le proporcione la clave para superar esa dualidad en su contexto diario, así como el bagaje desde el cual superar las escisiones del contexto filosófico. El propio Dewey en su obra Human Nature and Conduct expone como un amigo ${ }^{37}$ le hizo ver que una de las supersticiones actuales de las personas educadas era pensar que si a las personas se les dice que deben hacer y el fin que se busca es bueno, los resultados serán buenos ${ }^{38}$. Creencia mágica, según Dewey, que niega su atención a los significados que están implícitos para alcanzar un final. De hecho, Alexander, como Dewey señala ${ }^{39}$, destacó que la primacía de esta creencia, creaba nociones falsas sobre el control del cuerpo,

35 R. Shusterman, en este sentido, se ha hecho eco de esta reivindicación y ha desarrollado una nueva disciplina a partir del pragmatismo y la filosofía de John Dewey, denominada Somaesthetics, que conjuga teoría y práctica con una doble funcionalidad: por un lado enfatiza el papel perceptual del soma (entendido como cuerpo vivo que siente), por otro lado, despliega usos estéticos en la apreciación de las cualidades estéticas de nosotros mismos y las cosas. R. Shusterman la define así: "the critical, meliorative study of the experience and use of one's body as a locus of sensory-aesthetic appreciation (aisthesis) and creative self-fashioning" [Shusterman R., "Somaesthetics: A Disciplinary Proposal", en R. Shusterman, Pragmatist Aesthetics, cit., 265]. Véase también Shusterman, R., "Thinking through the body, educating for the humanities: A plea for somaesthetics". The journal of aesthetic education, $\mathrm{n}^{\circ}$ 40(1), 2006, pp. 1-21; Shusterman, R., Body consciousness: A philosophy of mindfulness and somaesthetics, Cambridge, Cambridge University Press, 2008; Shusterman, R., Thinking Through the Body, Cambridge, Cambridge University Press, 2012.

36 LW 3:29.

37 Este amigo es F. M. Alexander, tal y como reconoce en el artículo "Body and Mind" (1928). Asimismo, estudiosos de la obra de Dewey como Boydston (véase la conferencia pronunciada por él en el International Congress of Teachers of the Alexander Technique, 1986 (http://www.alexandercenter.com/jd/deweyalexanderboydston. html) o el capítulo II de la tesis doctoral de Erick McCormack, op. cit. Más recientemente destaca Shusterman R., "Redeeming Somatic Reflection: John Dewey", cit., pp. 180-216.

38 MW 14:27-28.

39 MW 14:28. 
olvidándose de la importancia del hábito controlado inteligentemente. En contraposición, para Dewey, al igual que para Alexander, la razón o inteligencia no es ajena a los hábitos, y proponer tal cosa es mera ficción ${ }^{40}$.

De este modo, reivindican una educación corporal que supere los hábitos asumidos y el fracaso de los remedios externos aplicados. Como señala el propio Dewey en el prefacio al libro de Alexander Constructive Conscious Control of the Individual, el método no es curativo, sino una educación constructiva y su campo de aplicación no son sólo adultos mal coordinados, sino que atañe también a la juventud o generación en desarrollo, para que desplieguen una apreciación sensorial y auto-juicio correctos.

\section{Bibliografía}

Alexander, Frederick Matthias (1918): Man's Supreme Inheritance, New York, E.P. Dutton and Company.

Alexander, Frederick Matthias (2000): The Universal Constant in Living, London, Mouritz. Alexander, Thommas M. (1987): John Dewey's Theory of Art, Experience and Nature: The Horizons of Feelings, Albany: SUNY Press.

Dewey, John (1969-1991), The Collected Works of John Dewey, Carbondale, Southern Illinois University Press.

Hickman, Larry. A. (ed.) (1992): The Correspondence of John Dewey, InteLex, versión electrónica.

Maisel, Edward (comp.) (1996): La técnica Alexander, Barcelona, Paidós.

McCullogh, Carol Porter (1996): The Alexander Technique and the String Pedagogy of Paul Rolland, Arizona, Arizona State University.

Shusterman, Richard (2008): Body consciousness: A philosophy of mindfulness and somaesthetics, Cambridge, Cambridge University Press.

Shusterman, Richard (2002): Pragmatist Aesthetics: Living Beauty, Rethinking Art, Oxford and Cambridge, Mass.: Blackwell.

Shusterman, Rirchard (2012): Thinking Through the Body, Cambridge, Cambridge University Press.

Tarr, Jennifer (2011): "Educating with the hands: working on the body/self in Alexander Technique", en C. McFarlane, R. L. Cohen y S. Nettleton, Sociology of Health and Illness Monographs: Body Work in Health and Social Care: Critical Themes, New Agendas, Malden, John Wiley \& Sons.

40 MW 14:25. 